\title{
A Novel Calibration Procedure that Insensitive to Stains and Colors for Pulsed TOF Cameras
}

\author{
Qiang $\mathrm{Wu}^{1 \mathrm{a}^{*}}$, Yincheng Liu ${ }^{1 \mathrm{~b}}$, Xuwen $\mathrm{Li}^{2 \mathrm{c}}$, Shinan Lang ${ }^{1 \mathrm{~d}}$ \\ ${ }^{1}$ College of Information and Communication Engineering, Faculty of Information \\ Technology,Beijing University of Technology, No.100 Ping Leyuan, Beijing, \\ P.R.China \\ ${ }^{2}$ College of Life Science and Bioengineering, Beijing University of Technology Beijing, No.100 \\ Ping Leyuan, Beijing, P.R.China \\ a wuqiang@bjut.edu.cn, ${ }^{b}$ liuyc91@qq.com, ${ }^{c}$ lixuwen@bjut.edu.cn, ${ }^{d}$ langshinan@bjut.edu.cn \\ *corresponding author
}

Keywords: TOF Camera, Depth Image, Calibration, Systematic Errors, Coordinate Transformation.

Abstract. The Time-of-Flight (TOF) camera is a new elegant choice for 3D measurement and modelling in many different fields. However, due to the different method of these new devices of the technology on the market, the knowledge of its performance is far away from enough. In particular, the distance measurements of these devices are often influenced by some systematic errors, which decrease the quality of the data a lot. To evaluate these errors, some experiments have been done on pulsed TOF cameras developed by our laboratory. In this paper, we proposed a novel calibration procedure for pulsed TOF cameras, which deal with the evaluation of the camera heating period, capture the intrinsic matrix of the camera, the measurement error of different distances and colors, and a quick transformation for coordinate.

\section{Introduction}

The Time-of-Flight (TOF) cameras provide a convenient and efficient access to capture depth information of real objects in real-time [1]. In this reason, these devices have an increasing use in 
image understanding, autonomous navigation, real-time modelling, and mapping. The TOF cameras emit pulsed or phased infrared illumination, and measure distances by the time of light emit and reflect back to the camera. T. Kahlmann [2] pointed out two different distance measurement methods as Figure 1.
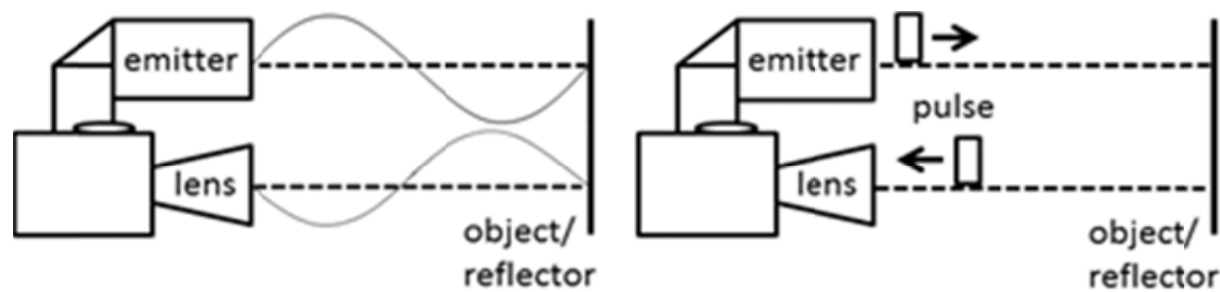

Figure 1 Time-of-flight distance measurement principle. Phase Shift (left) and Pulse Runtime (right).

For phased TOF cameras, each pixel on the TOF sensor can be measured through the distance to a single point of the object by means of a phase shift calculation. In contrast, the pulsed TOF cameras can directly calculate the time for a light pulse to travel back and forth to a point of the object at the distance.

Camera calibration is one of the most crucial and essential step for use of the cameras, and leads generally to a significant improvement of the precision of output. Several TOF calibration techniques have been proposed to remove the systematic TOF errors of phased TOF cameras, often in cooperation with the chosen of a specific error model. For instance, the distance errors are approached by B-splines in Lindner and Kolb [3], and in Kahlmann et al. [2], lookup tables have been used. Also, Fuchs et al. [4] determine parameters in a polynomial error model. But for pulsed TOF cameras, former works don't run that good because of the differences in measure method and resolution of the sensor.

Due to the use of high-resolution sensor and sensitivity to black color in middle process, pulsed TOF cameras can obtain intrinsic parameters by Zhang's method without assistant of another sensor, which is almost impossible for phased TOF cameras. In addition, in calibration procedure, a white wall or objects without any stains can hardly be found in most cases. Therefore, robust principal component analysis (RPCA) is introduced to reduce the impact of stains and colors. RPCA is a modification of the widely used statistical procedure of principal component analysis (PCA) which works well with respect to grossly corrupted observations. RPCA has many real life important applications particularly when the data under study can naturally be modelled as a low-rank plus a sparse contribution. And this is the first time when people use it in calibration procedure for pulsed TOF cameras as far as we know. Moreover, just like lateral calibration, it is the task for TOF cameras to map the camera pixels into a spatial reference frame. But the coordinate transformation is complex for real-time using. Thus, an efficient process should be carried out.

In this paper, a novel calibration procedure fulfil the above requirements is proposed. The procedure is systematic, reproducible as well as simple feasible. In Section 2, theoretical background of pulsed TOF cameras and RPCA is reviewed. In Section 3, the calibration procedure is laid out and illustrated 
with the pulsed TOF camera developed by ourselves. Section 4 shows some results and Section 5 finally concludes the paper.

\section{Theoretical Background}

\subsection{Measurement Method of Pulsed TOF Cameras}

Pulsed light operation is the most obvious method of operating a TOF system, because of its directly measured. The actual time measurement is performed by correlation of a start and stop signal with a parallel running counter.

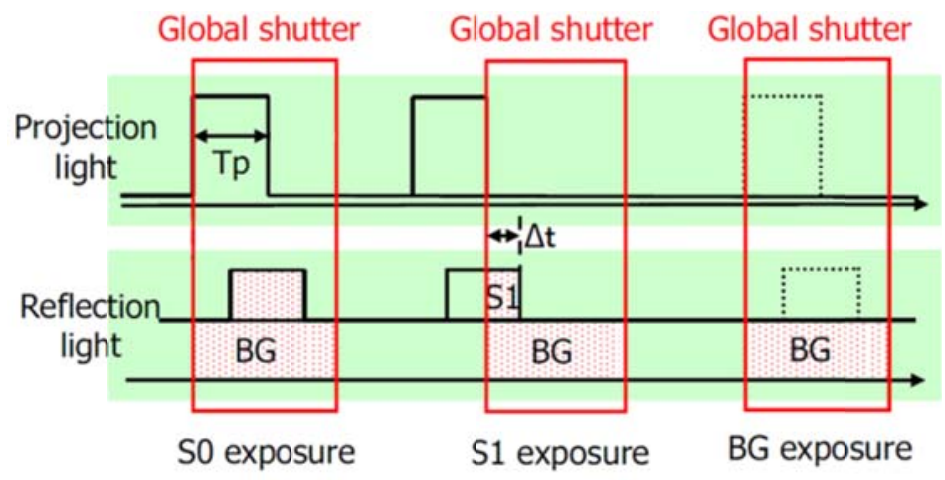

Figure 2 Method of pulsed TOF cameras.

As it shown in figure 2, the procedure as above cycles several thousand times, and we can read out the values in the sensor. Then the actual distance can be calculated by the values in the sensor. We can defined the speed of light as $\mathrm{c}$, while Tp represents the duration of the light pulse, and beyond this S0 stands for the collected charge of the earlier shutter and S1 for the charge with the delayed shutter, then for distance $\mathrm{d}$ the ratio is:

$$
\mathrm{d}=\frac{c \times T p}{2} \times \frac{S 1-B G}{S 0-B G}
$$

The largest measurable distance is measured if all charges in S1 and no charges at all in S0 are determined. The formula then yields:

$$
\mathrm{d}=\frac{c \times T p}{2}
$$

This is also an indicator that the light pulse width for this method determines the maximum measurable distance. 


\subsection{Algorithms of RPCA}

RPCA is a modification of widely used statistical procedure of principal component analysis (PCA) which works well with respect to grossly corrupted observations.

Suppose we are given a large data matrix $\mathrm{M}$, and we assume in the following:

$$
\mathbf{M}=\mathbf{L}_{0}+\boldsymbol{S}_{0}
$$

Where $\mathrm{L}_{0}$ has low-rank and $S_{0}$ is a small perturbation matrix.

Wright et al. [4] have proved that people can recover the low-rank matrix $\mathbf{L}_{0}$ from $\mathbf{M}=\mathbf{L}_{0}+\mathbf{S}_{0}$ by solving the following convex optimization problem

$$
\min _{L_{0}, S_{0}}\|\boldsymbol{L}\|_{*}+\lambda\|\boldsymbol{S}\|_{1} \quad \text { subj } \mathbf{L}+\boldsymbol{S}=\boldsymbol{M}
$$

The $\boldsymbol{L}$ and $\boldsymbol{S}$ here exactly recover the low-rank $\boldsymbol{L}_{0}$ and the sparse matrix $\boldsymbol{S}_{0}$.

RPCA optimization can be represented as

$$
\min _{X \in H} \boldsymbol{F}(X)=\mu \mathbf{g}(\mathrm{X})+\boldsymbol{f}(\mathrm{X})
$$

Where $\boldsymbol{f}(\mathbf{X}) \approx \frac{1}{2}\|\boldsymbol{A}(\boldsymbol{X})-\boldsymbol{b}\|^{2}$ is the penalty term of the equality constraint, $\mu>0$ and infinite approach to 0 . While $\boldsymbol{f}(\cdot)$ is a continuous convex function.

\section{The Proposed Calibration Procedure}

\subsection{Outline of the Proposed Calibration Procedure}

The proposed calibration procedure is outlined in Fig. 3 consisting of 4 steps, which are:

1. Camera heating. This step aims to reduce the impact of heat of semiconductors of the sensor.

2. Intrinsic calibration with S0 or S1 images. The purpose of this step is to capture the intrinsic matrix and correct the distortion of the lens.

3. Distance calibration with RPCA. It provides us with an access to catching precise depth data of pinhole model.

4. Transformation to spatial reference frame with cosine masks. We transfer pinhole model to spatial reference frame after this step. 


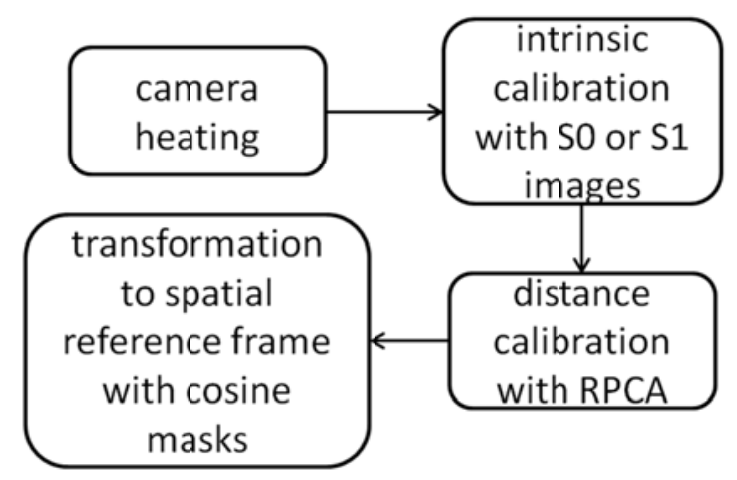

Figure 3 The outline of proposed calibration procedure.

\subsection{Camera Heating}

Materials of semiconductor materials are easily impacted by the change of both ambient temperature and heat of the camera. While ambient temperature is unpredictable, camera heating can be predicted and characterized by approach of O. Steiger [6].

\subsection{Intrinsic Camera Calibration}

For the pulsed TOF camera can be regarded as a pinhole model, we use Zhang's method [7] to obtain the intrinsic parameters of the camera. Zhang's method is popular in calibration, but due to the low-resolution sensor and measurement method of phased cameras, it can hardly work on them.

In this paper, we use Zhang's method and traditional checkerboard to assess the system error, in consistent with the intrinsic calibration. Though depth image as figure 4 shown can't be use in intrinsic calibration, pulsed TOF cameras provide us with distinct texture of the checkerboard in S0 or S1 image mentioned in sec. 2, so we can use S0 or S1 images shown in figure 5 to get the intrinsic parameters, and then the systematic measurement error and distortion can be corrected and removed.

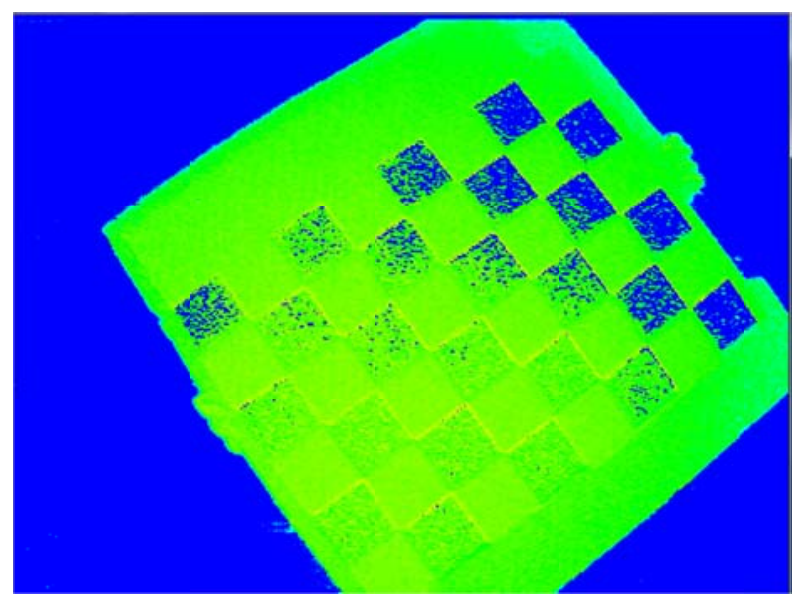

Figure 4 Depth image of the scene in pseudo-color. 


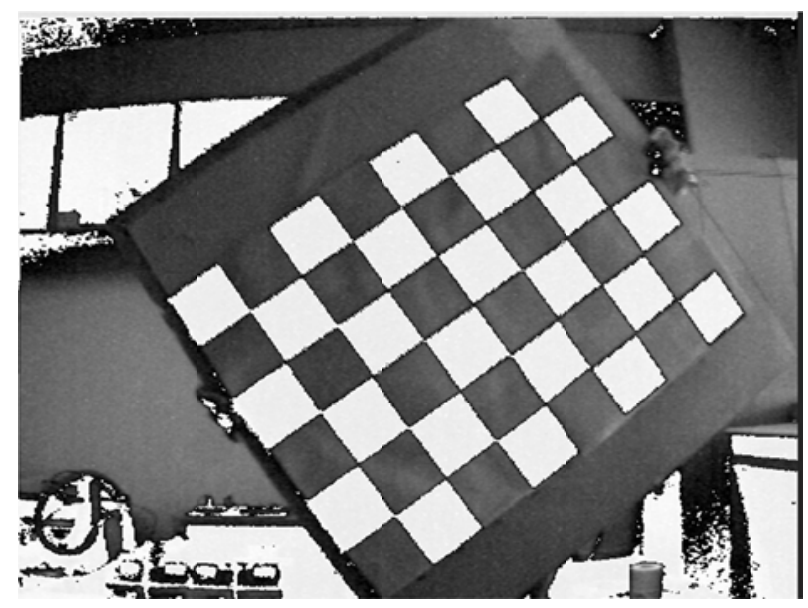

Figure 5 S1 image of the same scene.

\subsection{Distance Calibration with Robust Principal Component Analysis}

After intrinsic calibration of the pulsed TOF camera, the distance information is still unprecise and remains to be calibrated for better performance.

In method, the measured depths of pulsed TOF cameras conform to the linear characteristics with its distance from the object. In practice, however, it presented non-linear characteristics with its reference depth. Moreover, because the measurements of the center always shake in a small range and sometimes meet exposure error, we must find a way to get the precise value from sensor.

Noise come from exposure error are not Gaussian, but appear like spike, and always contains outliers. Also, impact of different colors is the same error model as well. In this phenomenon, the error model of the stains and different colors can be expressed by

$$
\mathbf{g}=\mathrm{Ku}+\xi
$$

Where $\mathbf{g}$ is depth image from the sensor, $\mathrm{K}$ is the operator of image resolution, $\boldsymbol{\xi}$ is sparse noise matrix, and $\mathbf{u}$ is the depth image to be recovered.

According to Lagrangian multiplier method

$$
\mathbf{J}(\mathbf{u})=\frac{1}{2}\|K \boldsymbol{u}-\boldsymbol{g}\|^{2}+\alpha \boldsymbol{J}_{T}(\boldsymbol{u})
$$

Where

$$
\boldsymbol{J}_{T}(\mathbf{u})=\int_{\boldsymbol{D}_{\boldsymbol{u}}} \sqrt{|\nabla \boldsymbol{u}|^{2}+\beta^{2}} d x d y=\int_{\boldsymbol{D}_{\boldsymbol{u}}} \sqrt{u_{x}^{2}+u_{y}^{2}+\beta^{2}} d x d y
$$

And value of $\alpha$ should be satisfy the constraint condition

$$
\min _{\mathbf{u}} \mathbf{J}_{\mathrm{T}}(\mathbf{u}) \quad \text { subj }\|\mathrm{Ku}-\mathbf{g}\|^{2}=\|\xi\|^{2}
$$

Where $\|\xi\|^{2}$ is the noise power of image $\boldsymbol{u}$. 
In this way, we used RPCA to get accuracy depth value and Look Up Table to correct the non-linear error and exposure error of the center pixel shown in figure 6 and then extended to every pixel.

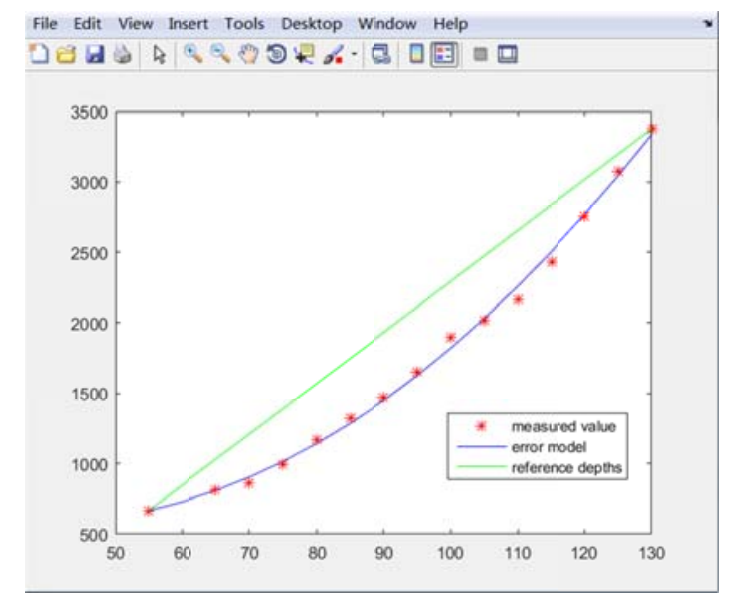

Figure 6 Non-linear characteristics with reference depths.

\subsection{Transformation of Coordinate}

The depth measurement accuracy for distance from every point of object to the center of the sensor has been investigated. However, the distance calibration for each pixel is not sufficient for utilization in reconstruction of object due to the pinhole model of the camera. In this way, a simple strategy to calculate the depth from each pixel to sensor plane is to acquire a cosine mask. This cosine mask contains an individual offset for every pixel. To gain this cosine mask, an accurate procedure was developed. A white wall and several reference distances $\mathrm{f}=45,50,55,60 \ldots, 145 \mathrm{~cm}$ was chosen, and we can measure each element of the cosine mask by following method.

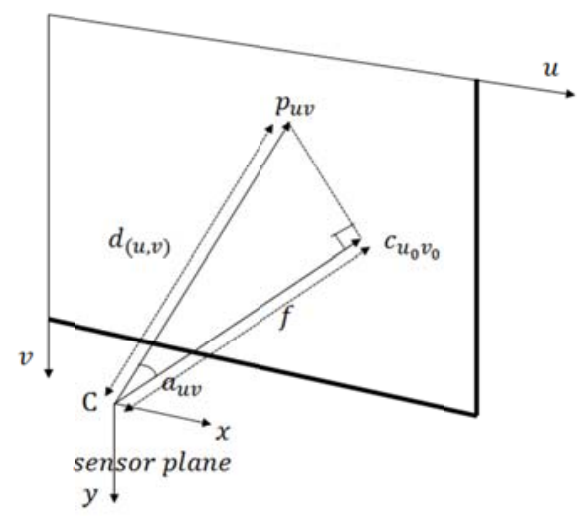

Figure $7 p_{u v}$ is the value of element of $P(u, v)$ in cosine mask 
As it shown in figure $7, \mathrm{p}_{\mathrm{uv}}$ is the value of element of $\mathrm{P}(\mathrm{u}, \mathrm{v})$ in cosine mask, and the value is computed by

$$
p_{u v}=\cos a_{u v}=\frac{d_{(u, v)}}{f}
$$

where $0 \leqslant \mathrm{u} \leqslant 640,0 \leqslant \mathrm{v} \leqslant 480$.

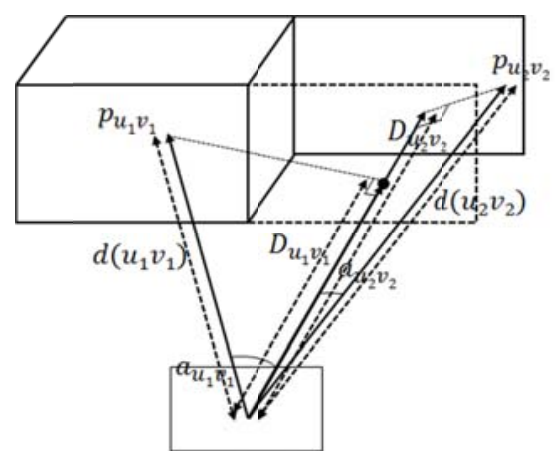

Figure 8 The distance of $d\left(u_{1} v_{1}\right)$ and $d\left(u_{2} v_{2}\right)$ can be calculated by cosine mask.

When confronted by other object, taking the object in figure 8 for an example, the depth $D_{u v}$ can be calculated by

$$
D_{u v}=\frac{d_{(u, v)}}{p_{u v}}
$$

\section{Results}

In our experiments, we used pulsed TOF camera developed by out laboratory, which has an opening angle of $23^{\circ} \times 15^{\circ}$ and a resolution of $640 \times 480$ pixel. As it shown in figure 9 , black velvet curtain has been used to reduce the error of multipath. In intrinsic calibration, we used a checkerboard pattern as a calibration object, printed out at a size of $54 \mathrm{~cm} \times 42 \mathrm{~cm}$ (black/white square size $6 \mathrm{~cm} \times 6 \mathrm{~cm}$ ). 

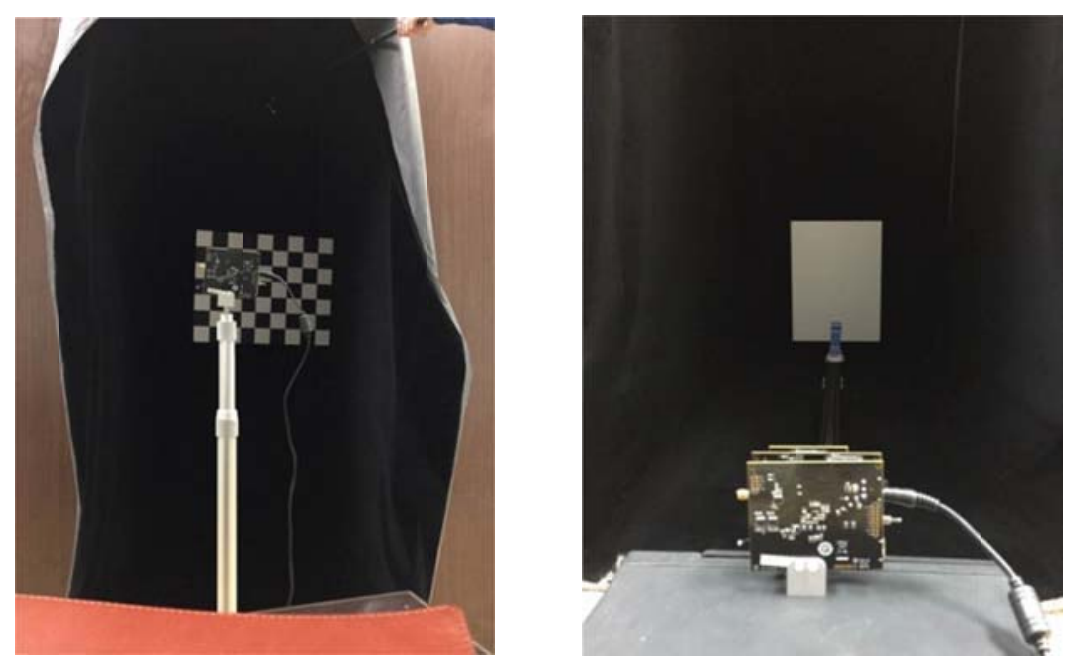

Figure 9 Setups of intrinsic calibration (left) and distance calibration (right).

We incorporated 32 images at different positions in front of the white panel and at different distances, covering a distance range of $0.8 \mathrm{~m}$ to $1.3 \mathrm{~m}$. As reference data for the calibration analysis, 10 images in the range of $0.65-1.45 \mathrm{~m}$ with a spacing of $5 \mathrm{~cm}$ have been carried out.

When confronted by white wall, we catch about 400 images in the range of $0.45-1.55 \mathrm{~m}$ with a spacing of $5 \mathrm{~cm}$ to calculate the cosine masks. Figure 10 illuminates the setups of the experiment without (left) and with (right) stains on the wall.
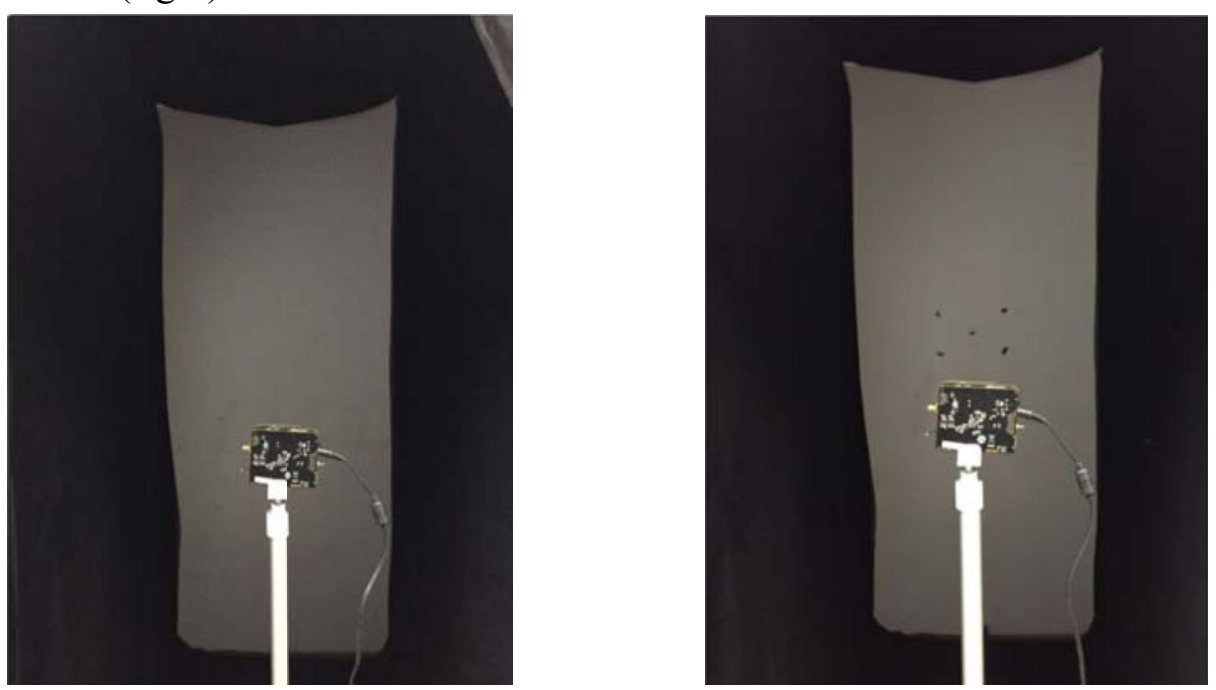

Figure 10 Setups of the experiment without (left) and with (right) stains on the wall.

Figure 11 shows the result of experiment without stains at distance of $95 \mathrm{~cm}$. Figure 11 (a) is the original data from TOF camera. We can see that margin area is bend. After removal of Fixed Pattern 
Noise (FPN), we can get figure 11 (b), where we can see the result is deviation from its reference value. Figure 11 (c) shows the result by cosine matrix, and the result is obviously better.

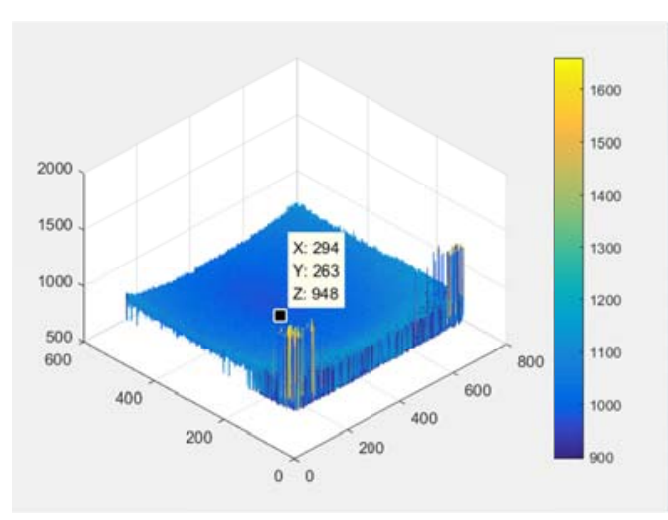

(a)

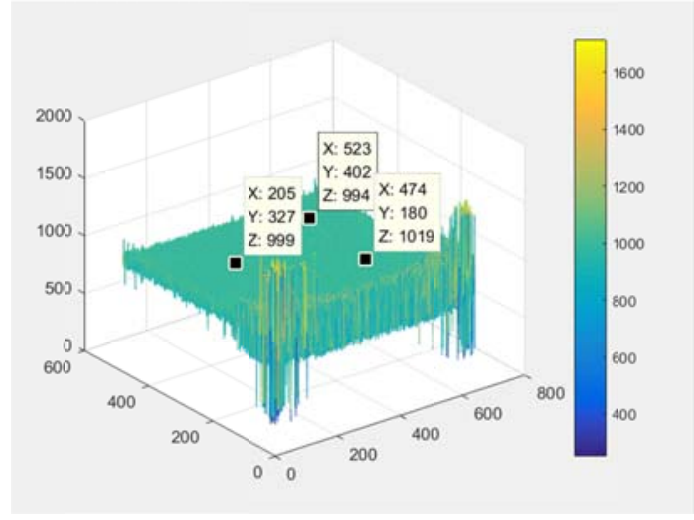

(b)

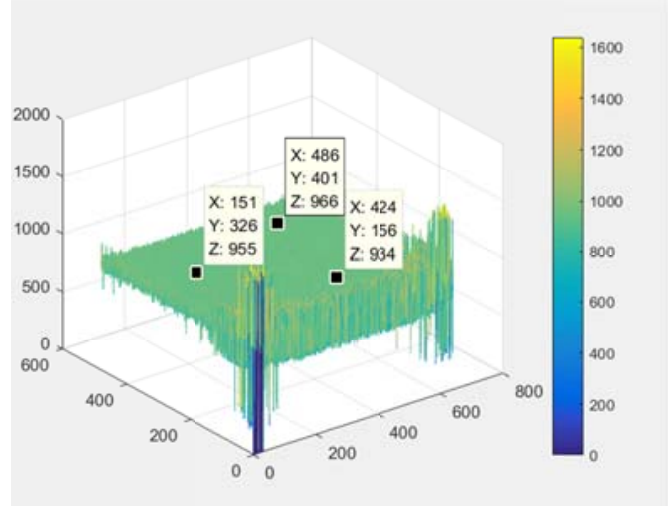

(c)

Figure 11 (a) is the original data from TOF camera, after removal of FPN we can get (b), (c) is the result by cosine masks.

Figure 12 shows the result of experiment with stains at distance of $95 \mathrm{~cm}$. Figure 12 (a) is the original data from TOF camera. We can see that depth deviation impacted by stains. And after FPN, we can get figure 12 (b), where we can see the noise is reduced but not entirely reduced by FPN. Figure 12 (c) shows the result recovered by RPCA, noise is absolutely removed. 


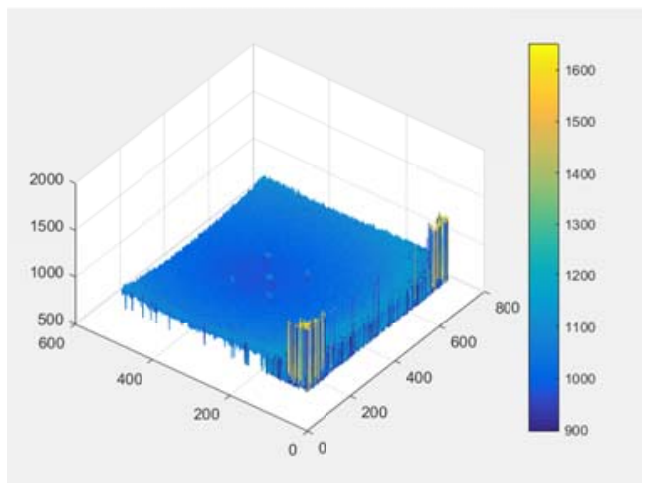

(a)

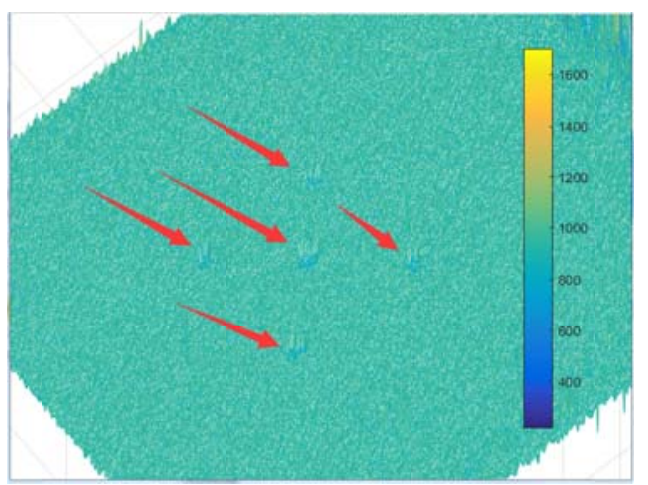

(b)

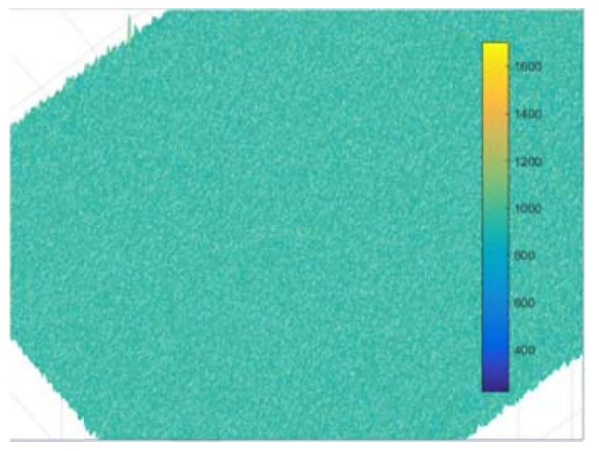

(c)

Figure 12 (a) is the original data from TOF camera, after removal of FPN we can get (b), (c) is the result by RPCA.

\section{Conclusions}

We have proposed and validated a novel calibration procedure which insensitive to stains and colors for pulsed TOF cameras, where we captured the intrinsic parameters by Zhang's method using S0 and S1 images. In addition, we the first made error model using RPCA then recovered the accurate depth image by correlation of look up table. Moreover, we come up with a quick transformation for coordinate by cosine masks. However, several issues have not yet been addressed. For instance, impacts of flying pixels were ignored. Also, influence factors of further or closer distances have not been considered. Nevertheless, the results are encouraging and the proposed calibration procedure is systematic, reproducible as well as simple feasible.

\section{References}

[1] Damien Leoch, Rahul Nair, Frank Lenzen, Henrik Schafer, Lee Streeter, Michael J. Cree, Reinhard Koch and Andreas Kolb, (2013) Technical Foundation and Calibration Methods for Time-of-Flight Cameras. 
[2] T. Kahlmann, F. Remondino, H. Ingensand, (2005) Calibration for Increased Accuracy of The Range Imaging Camera SWISSRANGERTM . Proc. SPIE Vol. 5665, 144-155.

[3] M. Lindner, A. Kolb, (2006)Lateral and depth calibration of PMD-distance sensors, International Symposium on Visual Computing (ISVC),, pp. 524 - 533.

[4] Emmanuel J. Candes; Xiaodong Li, Yi Ma, John Wright.(2009) Robust Principal Component Analysis?

[5] S. Fuchs, G. Hirzinger. (2008) Extrinsic and Depth Calibration of TOF-Cameras, Comp. Vis. Pattern Recognit. $1-6$.

[6] Olivier Steiger, Judith Felder, Stephan Weiss. (2010)Calibration of Time-of-Flight Range Imaging Cameras

[7] Z. Zhang. (2000)A Flexible New Technique for Camera Calibration. IEEE Trans. Pattern Anal. Mach. Intell., 22(11),1330-1334 